\title{
In Late-staged Atherosclerotic Foam Cells, Autophagy Exacerbates Lysosome Dysfunction and Cellular Homeostasis.
}

\author{
W. Gray Jerome ${ }^{1}$, Courtney Netherland-Van Dyke ${ }^{1}$ and Carrie Romer ${ }^{1}$ \\ 1. Department of Pathology, Microbiology and Immunology, Vanderbilt University, Nashville, USA
}

Autophagy is a key cellular homeostatic mechanism whereby internal contents of cells are targeted to lysosomes for digestion and reutilization. When autophagy does not function efficiently, pathology often occurs and so understanding what disrupts normal autophagy has become a key area of pathology research. During macroautophagy (hereafter referred to as autophagy), specialized double membranes are formed and surround the items to be targeted to lysosomes. This double-walled structure is referred to as an autophagosome. The autophagosome migrates to the lysosome and the autophagosome's outer membrane fuses with that of the lysosome membrane. This deposits the inner membrane and content into the lysosome lumen for digestion. In the last 5 years it has become clear that cellular lipid stores can be targeted by autophagy, and this lipid-specific form of autophagy has been termed lipophagy [1]. Microscopy has arisen as a key method for analyzing flux of material within the autophagy process [2].

Efficient lipid metabolism is critical to normal cell function and when disrupted can produce disease. Disruption of normal cholesterol metabolism can lead to the disease atherosclerosis where large amounts of cholesterol are stored in macrophages in the artery wall. These lipid-engorged macrophages (called foam cells because of their foamy appearance) are key contributors to the loss of normal arterial function that accompanies atherosclerosis progression. Most of the excess cholesterol in macrophages comes from the uptake of modified forms of low density lipoprotein (LDL) that carries cholesterol in the form of cholesteryl esters (cholesterol esterified to a long-chain fatty acid). Internalized LDL is delivered to lysosomes where the ester bond is cleaved and the resulting free cholesterol is transported out of the lysosome and used to regulate membrane function. However, when cholesterol is in excess, as it is in atherosclerosis, the macrophage cytoplasm can reesterify the cholesterol to a long-chain fatty acid and store the cholesterol in a cytoplasmic lipid droplets.

To get rid of cholesterol, cells must move the cholesterol to the plasma membrane where it can be transferred to a circulating lipid acceptor particle. Macrophages have cytoplasmic enzymes that can hydrolyze the cholesteryl esters in lipid droplets and move the generated free cholesterol to the plasma membrane. However, this process is inefficient. In contrast, lipophagy can target lipid droplet cholesteryl esters to lysosomes for hydrolysis, a more efficient process. Thus, enhancing autophagy has been proposed as a therapy for atherosclerosis to remove the stored cholesterol. However, we have shown that in late stage atherosclerosis, where lipid accumulation is extensive, cholesterol inhibits lysosome function $[3,4]$. Under these conditions, enhancing autophagy could exacerbate rather than relieve atherosclerosis lipid burden. In the current studies we investigated what occurs to lipophagy when lysosome function is disrupted by excess cholesterol. By comparing early foam cells, with functioning lysosomes to late stage foam cells where lysosome function is decreased we addressed several key questions. 1) In late stage macrophage foam cells does lipophagy continue? 2) In late stage foam cells does autophagy transport lipid to non-functioning lysosomes and thus contribute to overall lysosomal dysfunction and lysosomal sterol accumulation? 3) In macrophages with dysfunctional lysosomes, how does inhibition of autophagy effect overall sterol balance and macrophage function? 
We combined quantitative microscopy and biochemical approaches to analyze autophagic flux. To produce macrophages with various degrees of lysosome dysfunction (confirmed by microscopic lysosome $\mathrm{pH}$ measurement and biochemically determined cholesteryl ester hydrolysis), we incubated human macrophages with aggregated LDL. To assess the role of lipophagy in changes in cellular sterol metabolism, we compared lipid metabolism as lysosomes became more dysfunctional and also with identical cells in which autophagy was inhibited with vinblastin. Increases in lysosome dysfunction did not alter the constitutive rate of autophagy as measured by proteins critical to autophagosome assembly (Atg7, Atg5, p62, LC3) and visualization of autophagosomes. Moreover, autophagy targeted lipid droplet material to lysosomes as indicated by the progressive accumulation of perilipin 2 in lysosomes (figure 1) and a significant decrease in lysosome cholesteryl ester content when autophagy was inhibited. In cells with normal functioning lysosomes, inhibition of autophagy increased total cell sterol levels. However, in cells where lysosomal dysfunction was prominent, lysosomal levels of sterol decreased and efflux of cholesterol was slightly enhanced when autophagy was inhibited. Our data indicates that lipophagy continues in the face of lysosome dysfunction and autophagy-delivered cholesteryl ester can exacerbate overall lysosome sterol accumulation and macrophage dysfunction when foam cell sterol is excessive [5].

\section{References:}

[1] R Singh, et al., Autophagy regulates lipid metabolism. Nature, 2009. 458(7242): p. 1131-1135.

[2] D Klionsky, et al., Guidelines for the use and interpretation of assays for monitoring autophagy in higher eukaryotes. Autophagy, 2008. 4(2): p. 151-175.

[3] B Cox, et al., Effects of cellular cholesterol loading on macrophage foam cell lysosome acidification. J Lipid Res, 2007. 48: p. 1012-1021.

[4] W Jerome, et al., Lysosomal cholesterol accumulation inhibits subsequent hydrolysis of lipoprotein cholesteryl ester. Microsc Microanal, 2008. 14: p. 138-149.

[5] The authors acknowledge funding from NIH, NHLBI 5RO1HL86746 (WGJ), The American Heart Association Grant in Aid 15GRNT25700113 (WGJ), and The American Heart Association Postdoctoral Fellowship 13POST14240000 (CNV). Microscopy was supported in part by the VUMC Cell Imaging Shared Resource (supported by NIH grants CA68485, DK20593, DK58404, DK59637 and EY08126).
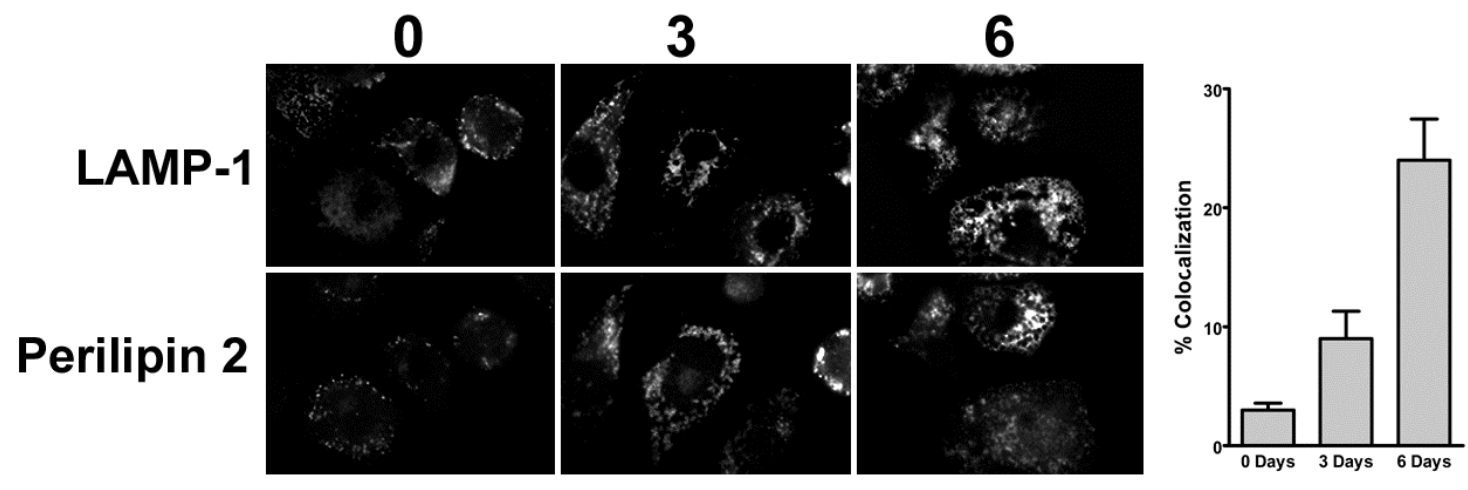

Fig 1. Macrophages were treated with aggregates of LDL for 0,3 or 6 days and immunostained for LAMP-1 (lysosome marker) and Perilipin 2 (lipid droplet marker). Colocalization of both perilipin with LAMP-1 indicates autophagy of lipid droplet material. 